\title{
A brain-machine interface instructed by direct intracortical microstimulation
}

\author{
Joseph E. O'Doherty 1,2, Mikhail A. Lebedev ${ }^{2,3}$,Timothy L. Hanson ${ }^{2,3}$, Nathan A. Fitzsimmons ${ }^{2,3}$ \\ and Miguel A. L. Nicolelis $1,2,3,4,5,6,7 *$
}

\author{
1 Department of Biomedical Engineering, Duke University, Durham, NC, USA \\ 2 Center for Neuroengineering, Duke University, Durham, NC, USA \\ ${ }^{3}$ Department of Neurobiology, Duke University, Durham, NC, USA \\ ${ }^{4}$ Department of Psychology and Neuroscience, Duke University, Durham, NC, USA \\ ${ }^{5}$ Edmond and Lily Safra International Institute of Neuroscience of Natal, Natal, Rio Grande do Norte, Brazil \\ ${ }^{6}$ Fellow, Center for Neuroprosthetics, Ecole Polytechnique Federale de Lausanne, Lausanne, Switzerland \\ 7 Blaise Pascal International Research Chair, École Supérieure de Physique et de Chimie Industrielles, Paris, France
}

\section{Edited by:}

Sidney A. Simon, Duke University, USA

Reviewed by:

Marshall Shuler, Johns Hopkins

University, USA

Rui M. Costa, Instituto Gulbenkian de

Ciência, Portugal

Eberhard E. Fetz,

University of Washington, USA

\section{*Correspondence:}

Miguel A. L. Nicolelis, Department of Neurobiology, Duke University, Box 3209, Room 327E Bryan Research Building, 101 Research Drive, Durham, NC 27710, USA.

e-mail: nicoleli@neuro.duke.edu
Brain-machine interfaces (BMIs) establish direct communication between the brain and artificial actuators. As such, they hold considerable promise for restoring mobility and communication in patients suffering from severe body paralysis. To achieve this end, future BMls must also provide a means for delivering sensory signals from the actuators back to the brain. Prosthetic sensation is needed so that neuroprostheses can be better perceived and controlled. Here we show that a direct intracortical input can be added to a BMI to instruct rhesus monkeys in choosing the direction of reaching movements generated by the BMI. Somatosensory instructions were provided to two monkeys operating the BMI using either: (a) vibrotactile stimulation of the monkey's hands or (b) multi-channel intracortical microstimulation (ICMS) delivered to the primary somatosensory cortex (S1) in one monkey and posterior parietal cortex (PP) in the other. Stimulus delivery was contingent on the position of the computer cursor: the monkey placed it in the center of the screen to receive machine-brain recursive input. After 2 weeks of training, the same level of proficiency in utilizing somatosensory information was achieved with ICMS of S1 as with the stimulus delivered to the hand skin. ICMS of PP was not effective. These results indicate that direct, bi-directional communication between the brain and neuroprosthetic devices can be achieved through the combination of chronic multi-electrode recording and microstimulation of S1. We propose that in the future, bidirectional BMIs incorporating ICMS may become an effective paradigm for sensorizing neuroprosthetic devices.

Keywords: brain-machine interface, primate, neuroprosthetics, cortical microstimulation, neuronal ensemble recordings

\section{INTRODUCTION}

During the last decade, considerable progress has been made in research on neuroprosthetics - devices that attempt to treat neurological impairments and limb loss by interfacing intact neural structures with artificial devices that enact motor or sensory functions (Nicolelis, 2003; Lebedev and Nicolelis, 2006; Schwartz et al., 2006; Fetz, 2007). Sensory neuroprosthetics, such as cochlear (Merzenich et al., 1974; Fallon et al., 2008) and visual (Dobelle et al., 1978; Dagnelie, 2008) implants, make use of electrical stimulation of sensory neural structures to recreate afferent input and perceptual experiences. Motor neuroprosthetics, often called BMIs (Nicolelis, 2001), transform neuronal activity recorded in motor areas into commands to move artificial actuators such as cursors (Serruya et al., 2002; Taylor et al., 2002) and robotic devices (Wessberg et al., 2000; Carmena et al., 2003; Velliste et al., 2008), and for the functional stimulation of muscles (Moritz et al., 2008).

Curiously, sensory and motor neuroprosthetics have developed largely in parallel, without attempts to build an integrated system with both motor and sensory capabilities. Normal interactions with the environment, however, require conjoint processing of sensory and motor signals (Witney et al., 2004; Crapse and Sommer, 2008). Thus, the neurophysiological mechanisms underlying voluntary motor control in mammals are critically dependent on the afferent innervation of the skin, muscles and joints (Ribot-Ciscar et al., 2003; James et al., 2007). Indeed, the paucity of sensory signals available in current BMIs could be a factor limiting their future clinical usefulness.

Here we propose that future clinical neuroprostheses could be based on a bi-directional system in which motor control signals are extracted from the brain, using multi-electrode arrays implanted in motor areas, while tactile, proprioceptive and other useful signals, are sent back to the brain through spatiotemporal patterns of intracortical microstimulation (ICMS) delivered to sensory areas (Lebedev and Nicolelis, 2006). To advance this goal, we added a direct intracortical input to a BMI, based on multi-electrode cortical microstimulation used by a rhesus monkey for enacting arm reaching movements (Figure 1A). This intracortical input instructed the direction of BMI-generated cursor movements. 


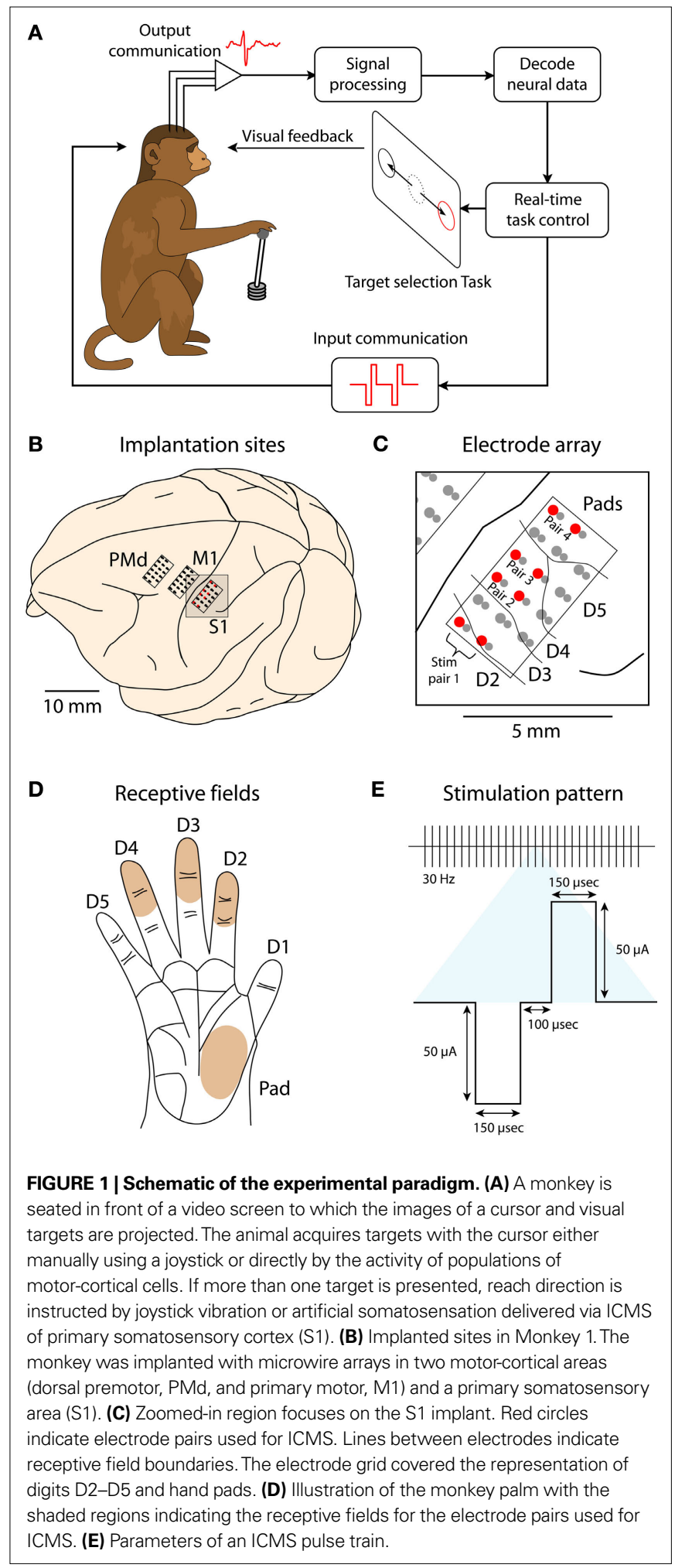

\section{MATERIALS AND METHODS SUBJECTS}

Two adult male rhesus macaque monkeys (Macaca mulatta, 6.7 and $6.5 \mathrm{~kg}$ ) participated in this study. All animal procedures were performed in accordance with the National Research Council's Guide for the Care and Use of Laboratory Animals and were approved by the Duke University Institutional Animal Care and Use Committee.

\section{CORTICAL IMPLANTS}

Each monkey received multiple microwire array implants in cortical areas, contralateral to their working hands. Aseptic surgery was performed to implant electrode arrays. Monkey 1 was implanted with six microelectrode arrays (32 microwires in each; Figures 1B,C) in primary motor cortex (M1), primary somatosensory cortex (S1) and dorsal premotor cortex (PMd) of both hemispheres. For the present study, we report only data from the arrays in the left hemisphere of Monkey 1. Monkey 2 was implanted with four 32-microwire arrays in the right hemisphere: M1, PMd, posterior parietal cortex (PP) and supplementary motor area (SMA).

Within each 32-channel array, electrodes were grouped into 16 pairs. Each pair consisted of two microwires positioned tightly together. One electrode in each pair was 300 microns longer than its mate, with the longer electrode always having equal or larger diameter. The separation between adjacent pairs was $1 \mathrm{~mm}$. Monkey 1 was implanted with polyimide coated tungsten electrodes, all 50 microns in diameter, in M1 and PMd and polyimide coated stainless steel 304 electrodes with 40 and 63 micron diameters in S1. Monkey 2 was implanted with arrays of mixed tungsten and stainless steel 304 electrodes in M1 and SMA. The tungsten electrodes were 50 microns in diameter and coated with polyimide insulation; the stainless steel 304 electrodes were 45 microns in diameter and coated with polytetrafluoroethylene. Monkey 2 also received polyimide coated tungsten electrodes, all 50 microns in diameter, in PMd and PP.

The PMd and M1 arrays were used to extract motor commands and the S1 array was employed as the target for ICMS (Figure 1C). In the second animal, motor signals were extracted from the SMA, PMd and M1 arrays, and ICMS was delivered to the PP array. The electrodes chosen for ICMS in Monkey 1 yielded recorded S1 neurons with clear receptive fields located on the ventral aspect of the second, third and fourth digits and palm pads (Figures 1C,D). No clear receptive fields were identified for the PP implant in Monkey 2. In this animal, we used the same arrangement of the stimulation electrodes within the electrode grid as described for Monkey 1.

\section{NEURONAL RECORDINGS}

Extracellular spiking activity was amplified, digitized, and highpass filtered using Multichannel Acquisition Processors (Plexon, Inc.). Action potentials were detected and sorted on-line with templates set by the experimenter using Plexon spike-sorting software. The quality of the units was quantified by analyzing the refractory period, estimated from the interspike intervals (ISIs). To be qualified as a single unit, in addition to having a distinct shape and amplitude (Nicolelis et al., 2003), at least $99.9 \%$ of its waveforms had to exhibit a refractory period greater than $1.6 \mathrm{~ms}$ (Hatsopoulos et al., 2004). Using these criteria $69.1 \%$ of the recorded units were single units, and $30.9 \%$ were classified as multi-unit neuronal activity. Single and multi-units were treated equally during online BMI sessions and for offline analysis purposes.

\section{BASIC EXPERIMENTAL PROCEDURES}

Each monkey was seated in a primate chair and viewed a computer display. Their heads were unrestrained and the recording 
system was connected to the implants using flexible wires. A two degree-of-freedom (left-right and forward-backwards) joystick was mounted at the monkey's waist level. The monkeys were trained to manipulate the joystick with their hands. Monkey 1 performed with the right hand; Monkey 2 performed with the left hand. An electrical touch sensor on the joystick handle measured whether the monkey was holding the joystick. An LCD projector displayed visual images on a screen mounted $1.5 \mathrm{~m}$ in front of the monkeys. Using the joystick, the monkeys moved a circular cursor with a 1.6- $\mathrm{cm}$ diameter. Forward, backward, rightward and leftward movements of the joystick translated to the upward, downward, rightward and leftward movements of the cursor, respectively. The joystick to cursor gain varied between 3.2 and 6.4 depending on the session (i.e., a 1-cm movement of the joystick translated into a 3.2 - to $6.4-\mathrm{cm}$ movement of the cursor). Targets were defined by rings 4.8 to $5.6 \mathrm{~cm}$ in diameter.

\section{BEHAVIORAL TASKS}

Three behavioral tasks were employed (Figures 2A-C). The first two tasks, center-out (Figure 2A) and continuous target pursuit (Figure 2B), served to estimate basic parameters of the BMI such as prediction accuracy and neuronal tuning to motor parameters. The third task, target choice (Figure 2C), incorporated somatosensory inputs. Each task required placing the computer cursor into a target using the joystick. Correct performance was rewarded by juice rewards. The center-out task used stationary targets that occurred at randomly chosen locations on a fixed-radius from the center of the screen. The monkey was required to hold the cursor at the central target and, once a peripheral target appeared, move the cursor to that target.

The pursuit task used a moving target that followed a Lissajous curve as described by:

$x=A \sin (a v t+\delta)$

$y=B \sin (b v t)$

where $x$ and $y$ are the $x$ - and $y$-axis coordinates and $t$ is time in milliseconds. We used parameter values $a=3, b=4, v \in\{0.00015$, $0.0002\} \mathrm{kHz}, \delta=0.5 \pi$, and $A=B=22.4 \mathrm{~cm}$.

The target choice task started with the monkey centering the cursor at a target that appeared in the middle of the screen. Then, an instruction period of $0.5-2$ s followed. During this interval, a somatosensory stimulus was presented that instructed reach direction. In some experimental sessions, the stimulus instructed reaching to the right; in the others it instructed reaching to the left. If no stimulus was delivered, the monkey had to reach to the opposite direction. The stimulus itself was either a vibration of the joystick handle or ICMS. In some sessions, the stimulus was delivered for the entire instruction interval; in others, it was delivered for a fraction of the total instruction interval. After the instruction period ended, the central target disappeared and two choice targets appeared on the screen. The monkey was rewarded for responding by placing the cursor over the target (right or left) corresponding to the direction indicated by the stimulus delivered during the instruction interval.

In some sessions, we repeated the same stimulus in the trial immediately following one in which the monkey made an error. This was done for training purposes to prevent the monkeys from choosing the same target repeatedly without ever making reaches to the other target. However, when this rule was employed the monkeys could potentially make correct reaches in these trials without attending to the stimulus. Therefore, in sessions where this technique was employed, we restricted our analysis to trials that followed a rewarded trial and excluded those that followed non-rewarded trials.

\section{INTRACORTICAL MICROSTIMULATION}

Electrical stimulation was accomplished with a custom-built 4-channel current-controlled, optically isolated stimulator. Simulation trains used in this study consisted of symmetric, biphasic, charge-balanced waveforms delivered synchronously and in a bipolar fashion through adjacent pairs of electrodes. Current amplitudes of 25-60 $\mu \mathrm{A}$ and pulse widths of 150-200 $\mu$ s were used at frequencies between 30 and $60 \mathrm{~Hz}$, depending on the session (Figure 1E). The initial current amplitudes used for ICMS of S1 were determined by first finding the threshold current for evoked movements in M1 and then selecting a value just below that threshold. There was a 100- $\mu$ s delay between the cathodic and anodic phases of the stimulation waveforms due to limitations with the stimulation hardware.

\section{LINEAR DISCRIMINANT ANALYSIS}

To classify reach direction during the instruction period of the target choice task, we used linear discriminant analysis (Fisher, 1936). The interval from $1 \mathrm{~s}$ prior to the onset of the instruction period to $1 \mathrm{~s}$ after the offset was broken into $50 \mathrm{~ms}$ bins. The neuronal firing rate for each of the neurons in the ensemble was placed into a vector for each time-bin. This procedure was repeated for each trial. The trials were then partitioned into those in which the reach was made to the right and those where the reach was made to the left. From each of these sets, half of the trials were selected for training the classifier and half were selected for cross-validation. Using equal numbers of trials from each training set, linear classifiers were trained to discriminate reaches to the left and to the right for each time-bin. These classifiers, however, acted causally and cumulatively based on information accumulated from previous time-bins. This means that the classifier at time $t=T$ used the output of the preceding classifiers $(t<T)$ as well as the firing rates of the neurons at time $t=T$ to classify reach direction. Performance of the classifier was measured with the cross-validation set.

\section{PREDICTION ALGORITHM}

Cursor position was reconstructed using multiple Wiener filter linear decoding algorithms applied to the population of recorded neurons (Wessberg et al., 2000; Haykin, 2002; Carmena et al., 2003; Lebedev et al., 2005). The filter represented the $X$ and $Y$ coordinates of the joystick as a weighted sum of neuronal firing rates measured before the time of decoding:

$X(t)=b+\sum_{i=1}^{N} \sum_{j=1}^{10} w_{i j} n_{i}[t-(j-1) \tau]+\varepsilon(t)$

where $X(t)$ is the value of the decoded parameter (for example, $X$-coordinate of the joystick) at time $t, n_{i}$ is the firing rate of neuron 


\section{A Center out task}

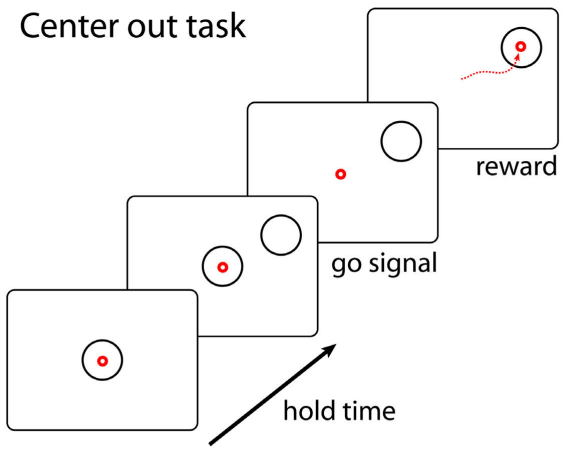

B

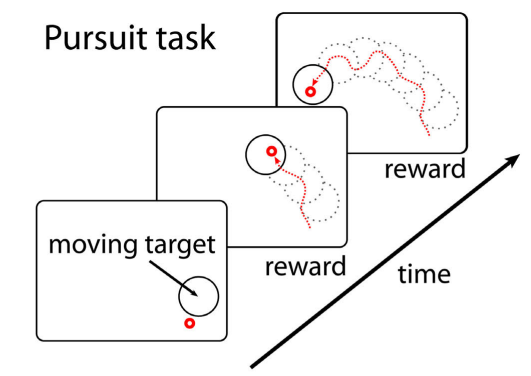

C Target choice task

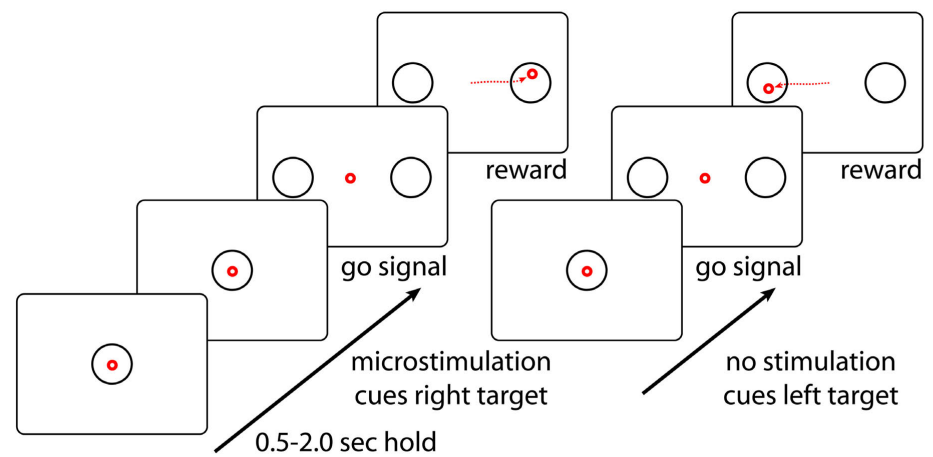

D Example traces

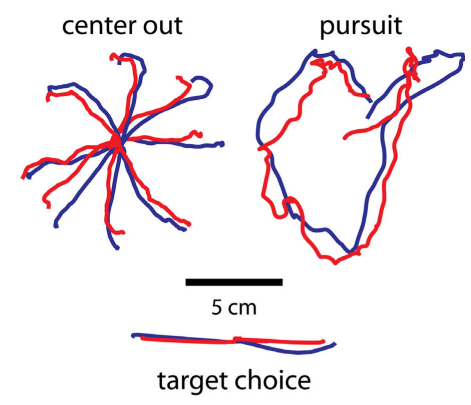

E

$$
\begin{aligned}
& \sim-v-\sqrt{-N} \\
& \leftrightarrow r \sqrt{n} \sim \sim r
\end{aligned}
$$

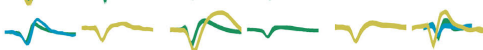

$$
\begin{aligned}
& \text { arr } r-r-r
\end{aligned}
$$

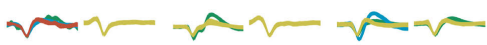

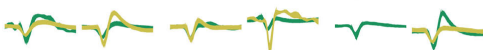

$$
\begin{aligned}
& \sim \sim \sim \sim \sim \sim r .
\end{aligned}
$$

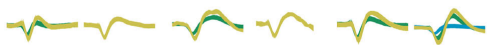

$$
\begin{aligned}
& \text { arv }-\sqrt{-r}-r
\end{aligned}
$$

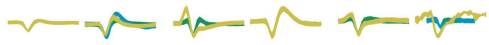

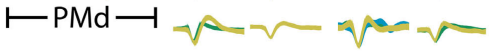

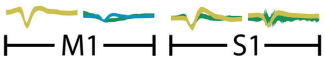

FIGURE 2 |The three reaching tasks performed manually and through the BMI. (A) Center-out task. Monkeys first placed the cursor at a central location and then moved it to eccentrically located targets. (B) Continuous target pursuit task. Monkeys were rewarded for keeping the cursor within a continuously moving target. (C) Target choice task. Monkeys selected one of two targets. Directional instruction was provided by joystick vibration or intracortical microstimulation (ICMS) of S1. (D) Example traces for each of the tasks. Blue

$\mathbf{F}$

\section{Neuronal tuning}

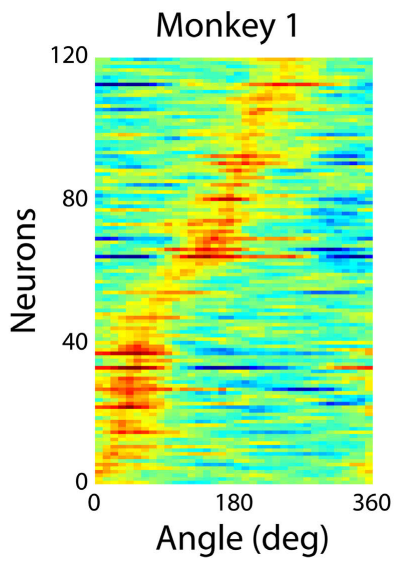

Monkey 2
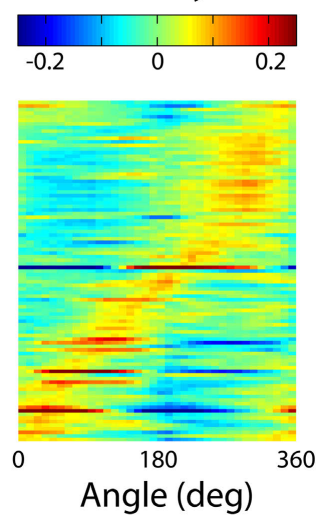

traces are hand movements; red traces are predictions made from the neuronal ensemble activity. (E) Neuronal waveforms simultaneously recorded in three cortical areas (PMd, M1 and S1). (F) Directional tuning curves for the ensembles of cortical neurons recorded in Monkeys 1 (left) and 2 (right). Each horizontal line represents a neuron. Color represents firing rate normalized by standard deviation as a function of movement direction; neurons are sorted by preferred direction. $i, N$ is the total number of neurons, $(j-1) \tau$ is the time delay for tap $j, w_{i j}$ is the weight for neuron $i$ and tap $j, b$ is the $y$-intercept and $\varepsilon(t)$ is the residual error. The word tap here simply refers to a time delay between the input (neuronal firing rate) and output (joystick movement) of the filter. The value of the tap length parameter $\tau$ was set to $100 \mathrm{~ms}$, and the number of taps was set to 10 , that is neuronal rates were sampled in a 1000 -ms window preceding the time of decoding. 

form:

To calculate model weights, $w$, Eq. 2 was converted to matrix

$\mathbf{X}=\mathbf{N W}+\varepsilon$

where $\mathbf{X}$ is the matrix of joystick movements, $\mathbf{N}$ is the matrix of neuronal rates, $\mathbf{W}$ contains the weights of the model, $\varepsilon$ is the error. Each row of $\mathbf{N}$ corresponds to a specific time and each column is a vector of data for a particular neuron and time lag. Since our models took into account 10 lags, matrix $\mathbf{N}$ had 10 columns for every neuron. The $y$-intercept was calculated from a column of ones prepended to matrix $\mathbf{N}$. We then solved for matrix $\mathbf{W}$ using the linear least squares approximation:

$\mathbf{W}=\operatorname{inv}\left(\mathbf{N}^{T} \mathbf{N}\right) \mathbf{N}^{T} \mathbf{X}$

Each filter was first trained (i.e., the values of weights $\mathbf{W}$ were calculated) and then used as the decoder for new data. Accordingly, each experimental record was split into two halves: the training set and the validation set. The model was trained on the first set of the experimental data and predictions were obtained using the second set. Decoding was also conducted for the reverse arrangement: training the models on the second set and using it to predict the first set.

\section{DIRECT BRAIN CONTROL}

For each of the tasks, online, closed-loop experiments were performed. The monkeys first performed the behavioral tasks manually using a hand-held joystick (Hand Control; HC) and later controlled the cursor movements directly with their cortical activity. The behavioral and neural data, recorded during 10-30 min training epochs during which the monkeys moved the joystick manually, were used to train multiple linear decoding models that extracted cursor position from the modulations of cortical cells, as described above. Once the model weights were calculated, the mode of operation was switched to brain control during which the joystick was disconnected from the cursor, and cursor position was directly controlled by the signals extracted from the brain. For some sessions the joystick was immediately removed upon transition to brain control (Brain Control Without Hand movements; BCWOH); for other sessions, the joystick was disconnected from the task control but otherwise left alone and the monkey was permitted to continue to move its hand (Brain Control With Hand movements; BCWH). For still other sessions, brain control was performed in both $\mathrm{BCWH}$ and $\mathrm{BCWOH}$ modes.

\section{RESULTS}

In this study, a somatosensory component was added to our previous design of a BMI enacting arm reaching movements (Carmena et al., 2003). This component instructed the direction of reaching movements. Motor output of the BMI was controlled by the activity of neuronal ensembles recorded in the primary motor (M1) and dorsal premotor (PMd) cortex (Figure 1B). The behavioral tasks consisted of acquiring visual targets with a computer cursor (Figures 1A and 2). Monkeys first performed the behavioral tasks manually using a hand-held joystick and then switched to controlling the cursor directly by their cortical activity. Predictions of cursor movement were generated using linear models (Wessberg et al., 2000; Carmena et al., 2003). Two types of somatosensory input were employed: (a) vibrotactile stimulation of the monkeys' palms via a vibrator embedded in the joystick handle and (b) ICMS of primary somatosensory (S1) cortex in one monkey (Figure 1E) and the arm reach area of posterior parietal cortex (PP) in the other monkey. Both monkeys learned to use vibrotactile stimulation cues to instruct the direction of their cursor movements. The first monkey learned manual and BMI tasks instructed by ICMS of S1; the other was unable to learn to use ICMS of PP.

\section{ENSEMBLE ACTIVITY DURING REACHING TASKS}

We recorded simultaneously from ensembles of 50-200 cortical neurons, depending on the experimental session and subject (Figure 2E). Analysis of directional tuning indicated that the preferred directions of the recorded neurons covered the entire range of angles from 0 to $360^{\circ}$ (Figure 2F). Concurrent with recordings during behavioral tasks, we performed periodic mapping of receptive fields of the neurons recorded in S1. These neurons revealed receptive fields distributed primarily on the hand and digits (Figures 1C,D).

\section{DECODING MOVEMENT}

The accuracy of predictions of hand position from cortical ensemble activity observed was in the same range as has been achieved in previous studies carried out in our laboratory (Wessberg et al., 2000) and by other groups (Taylor et al., 2002). The prediction accuracy was higher in the early recording sessions, when the majority of implanted electrodes yielded the most high quality units and later decreased as some of the channels lost unit activity. Table 1 shows the average performance of the predictions under different conditions. Across all tasks and sessions, when performing in $\mathrm{HC}$ mode, the average correlation coefficient $(\mathrm{R})$, between the actual and predicted values of the joystick X-position, was $0.47 \pm 0.19$ (mean \pm standard deviation) for Monkey 1 and $0.53 \pm 0.14$ for Monkey 2. The R for Y-position was $0.42 \pm 0.18$ and $0.51 \pm 0.17$ for Monkeys 1 and 2, respectively.

Table 1 | Average prediction accuracy. Correlation coefficient between the actual hand movements and movements as predicted from cortical activity across different behavioral conditions for two monkeys. Sessions with brain control with hand movements $(\mathrm{BCWH})$ are shown split into the initial hand control portion, when the online filter was trained, and the subsequent brain control portion. The mean \pm standard deviation is presented.

\begin{tabular}{lll}
\hline Session type & Monkey 1 & Monkey 2 \\
\hline HC & $\boldsymbol{N}$ (sessions) = 139 & $\mathbf{N = \mathbf { 7 8 }}$ \\
\hline$x$ & $0.47 \pm 0.19$ & $0.53 \pm 0.14$ \\
$y$ & $0.42 \pm 0.18$ & $0.51 \pm 0.17$ \\
\hline BCWH & $\boldsymbol{N}=\mathbf{2 0}$ & $\mathbf{N = \mathbf { 2 1 }}$
\end{tabular}

Hand control portion

$x$

$y$

$0.57 \pm 0.20$

$0.58 \pm 0.14$

Brain control portion

$x$

$0.60 \pm 0.20$

$0.45 \pm 0.19$

$0.41 \pm 0.19$

$0.48 \pm 0.16$ 
The correspondence between the actual and predicted hand position decreased in sessions where the monkeys were permitted to continue to move their hands during direct brain control (BCWH; Table 1). The R for X-position decreased 28.1\% $(P<0.01$ Wilcoxon signed-rank test) and $17.2 \%$ in Monkey $2(P<0.001)$. The $\mathrm{R}$ for Y-position decreased 16.7\% $(P<0.01)$ and $15.6 \%$ $(P<0.005)$ in Monkeys 1 and 2, respectively. This decrease indicates that the neuronal ensemble adapted to controlling the cursor movements and became less representative of the animal's hand movements - a finding that we and others have previously interpreted as evidence of the external actuator becoming incorporated into the brain's internal representation (Lebedev et al., 2005; Tkach et al., 2007).

\section{SOMATOSENSORY STIMULUS}

Incorporation of somatosensory input into the BMI was investigated in the target choice task (Figure 2C). The monkeys chose between two visually identical targets based on a somatosensory input (vibration or ICMS), the presence or absence of which instructed reach direction (right or left identical circles; Figure 2C). In initial experiments with Monkey 1 , the presence of a stimulus instructed reaches to the right, and the absence of stimulus instructed reaches to the left. This contingency was later reversed. Monkey 2 reached to the left if the stimulus was presented and reached to the right if it was absent.

Monkey 1 was initially trained with vibrotactile stimulation and was able to perform at $90 \%$ accuracy after 12 sessions (Figure 3A). After 15 sessions of training in this task, the vibration cue was replaced with ICMS delivered to S1. However, the previous training with vibrotactile stimulation did not immediately transfer to the ICMS task. Rather, the monkey started with chance performance and began to improve only on the 15th session. After this point, learning was very rapid and the monkey achieved 90\% accuracy by the 20th session. (Figure 3B).

However, even before rapid behavioral improvement occurred in the ICMS task, the monkey's performance was marginally, but statistically, above chance $(60.02 \%$ versus $52.5 \%$ in control trials without microstimulation; $P<0.001$, Wilcoxon ranked-sum test). This effect could reflect a bias in the monkey's reach decision that resulted from the activation of motor preparatory circuits in the stimulated hemisphere (Hanks et al., 2006). If so, this bias was not crucial for task performance because the monkey learned a reverse contingency in which the instructed target was ipsilateral to the stimulated hemisphere (Figure 3C).

Psychometric measurements in which ICMS current amplitude was varied on different trials showed that Monkey 1 could respond to currents as low as $25 \mu \mathrm{A}$ (with a pulse width of $150 \mu \mathrm{s}$ ) and reached $90 \%$ accuracy when ICMS amplitude was at $50 \mu \mathrm{A}$ (Figure 3D). The average proportion of correctly performed trials with an ICMS amplitude greater than $20 \mu \mathrm{A}$ was $88 \%$, which was significantly different from chance $(P<0.001$, Wilcoxon rankedsum test). For currents less than $20 \mu \mathrm{A}$ the monkey performed at $50 \%$ chance level $(P=0.8)$. Curiously, Monkey 1 exhibited better performance when ICMS amplitude exceeded $20 \mu \mathrm{A}$ in the trials immediately following error trials in which the instruction simply repeated. In these trials, the monkey could receive a reward by simply changing the previous erroneous direction, so the instruction, in principle, could be ignored. Nonetheless, for ICMS currents less than $20 \mu \mathrm{A}$ Monkey 1 performed correctly in only $72 \%$ of trials, which was significantly different from chance $(P<0.001)$, but significantly worse $(P<0.001)$ than the $84 \%$ correct performance with ICMS currents greater than $20 \mu \mathrm{A}$. Thus, the ICMS cue clearly benefited performance even in those trials. This effect was also clear from an analysis of reaction times. The reaction time on regular trials (i.e., rewarded trials preceded by rewarded trials) was $457 \pm 43 \mathrm{~ms}$ (mean \pm standard error) for the currents less than $20 \mu \mathrm{A}$ and $454 \pm 18 \mathrm{~ms}$ for the higher currents. The small $3 \mathrm{~ms}$ average difference was statistically significant $(P<0.001$, Wilcoxon ranked-sum test). In the trials following error trials, the monkey's decisions occurred significantly slower than on regular trials $(P<0.001)$, as indicated by increased reaction times: $785 \pm 60 \mathrm{~ms}$ for currents less than $20 \mu \mathrm{A}$ and $562 \pm 51$ for those higher. Thus, higher ICMS currents resulted in a considerable $(223-\mathrm{ms} ; P<0.001)$ improvement in the reaction time.

Monkey 2 learned the vibrotactile cued task on a similar timecourse as Monkey 1 ( 7 sessions to start learning and 11 sessions to reach $>90 \%$ accuracy; Figure $3 \mathrm{~A}$ ). This animal did not have an implant in $\mathrm{S} 1$ and was unable to learn the task instructed by ICMS of the PP even though training with ICMS continued for 30 sessions.

\section{BMI WITH SOMATOSENSORY INPUT}

In the BMI with somatosensory input, one monkey controlled cursor movements directly by using motor cortical activity while receiving somatosensory instructive signals in the form of $\mathrm{S} 1$ ICMS. The second monkey also controlled the cursor using motor cortical activity but, since PP ICMS was ineffective, received somatosensory signals via vibrotactile stimulation of the hand. The major technical challenge for carrying out these experiments was obtaining simultaneous recordings from large neuronal populations in motor cortical areas concurrent with ICMS to sensory cortical areas. The potential for ICMS pulses occluding spikes was limited to $6-12 \%$ of the stimulation interval because electrical artifacts evoked by the pulses were brief $(<2 \mathrm{~ms})$ and easily distinguishable from action potentials. While we recorded neuronal activity during ICMS, we excluded the instruction intervals from the dataset used to train the BMI algorithms. This was done to avoid any influence of the stimulation on the parameters of the extraction algorithm (e.g., occluded spikes interpreted as a decrease in firing rate or neuronal responses to ICMS confused with motor commands). No neuronal activity was excluded during online BMI operations.

Neuronal modulations were observed both during the instruction and movement periods of the target choice task. We observed neurons that exhibited increases and decreases in their firing rates during the instruction period, as well as neurons that were directionally selective exclusively during the movement period (Figure 3E). Movement direction could be predicted from neuronal population activity before the monkey moved the joystick (Figure 3F). In the early trials, when the monkey performed close to chance level (blue trace), the rise in the predictions occurred before the delay. This suggests that the monkey started to form its decision before the stimulus was delivered. 

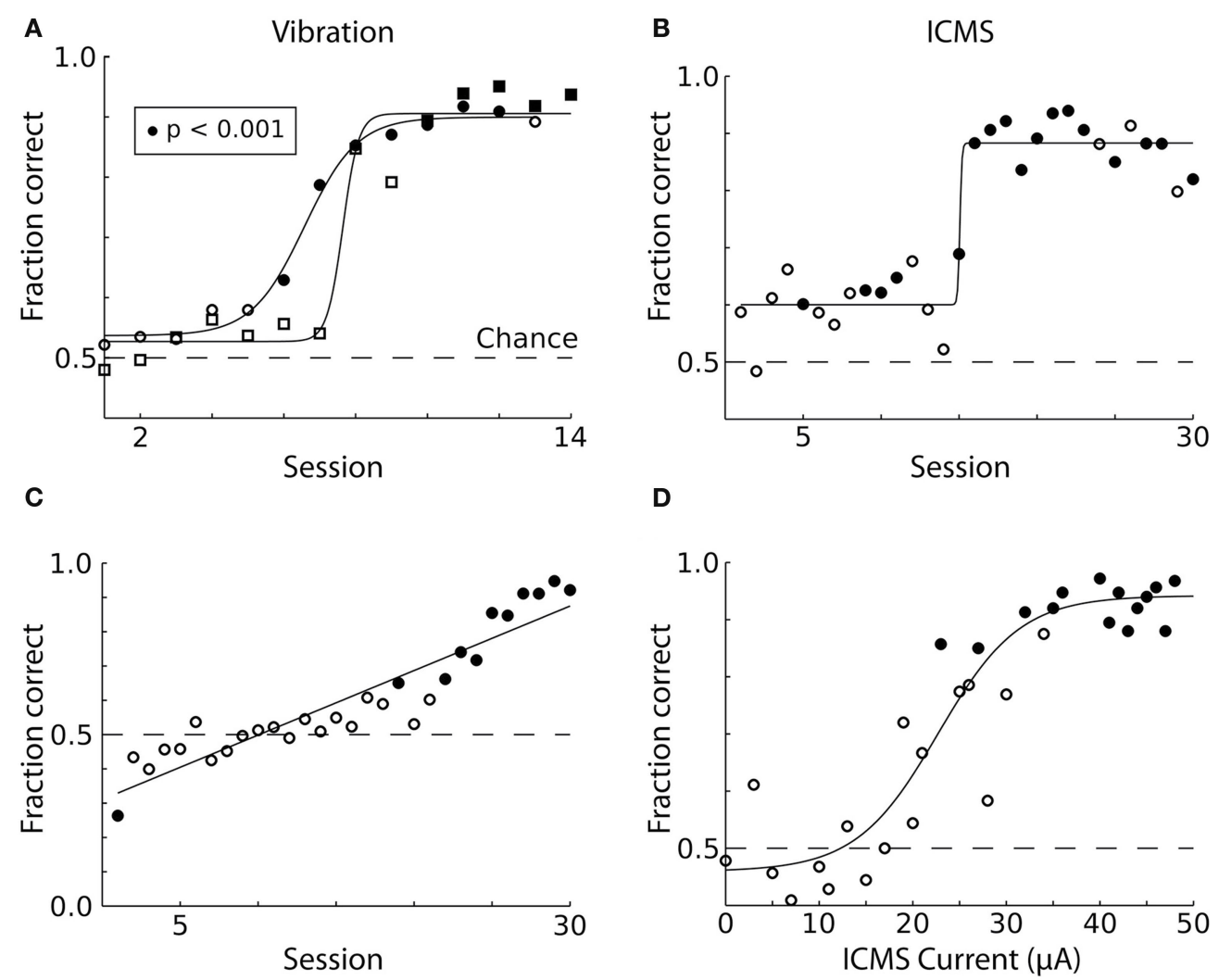

D
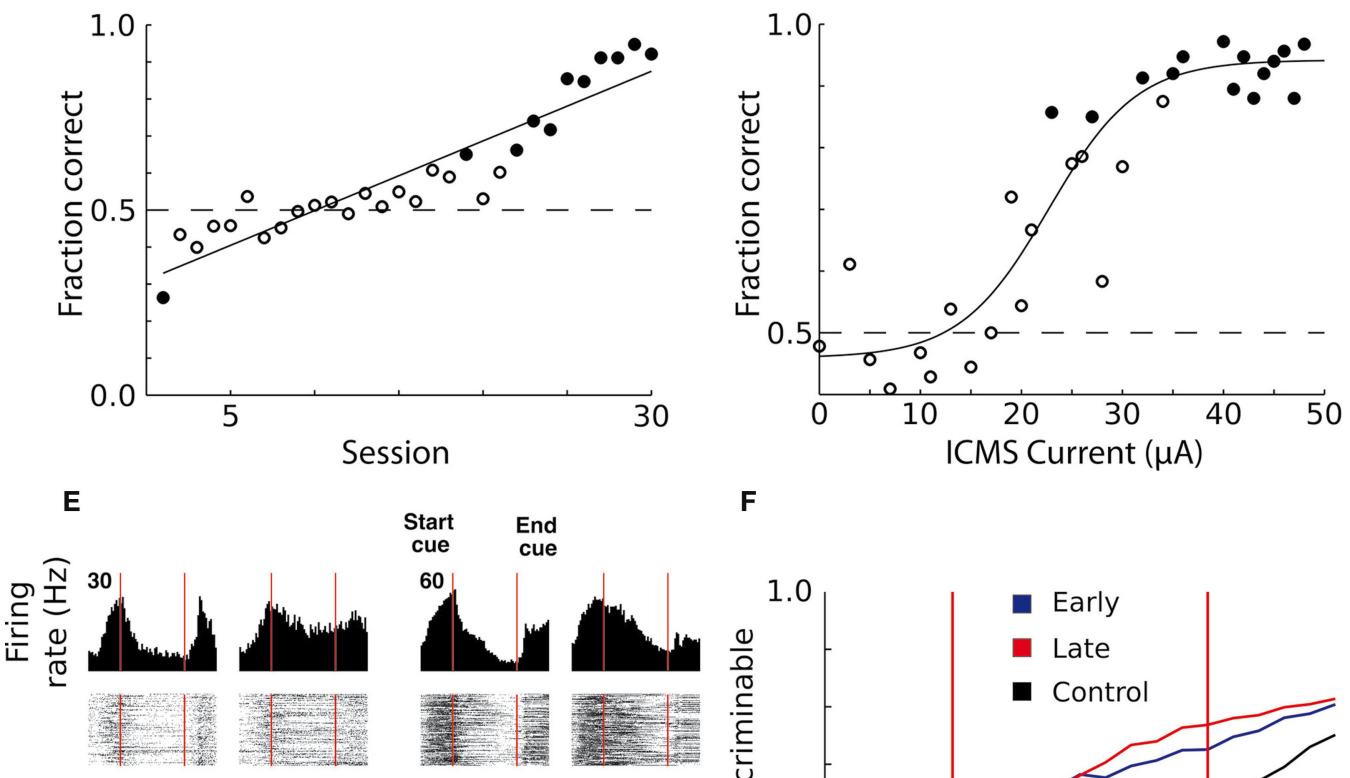

$\mathbf{F}$

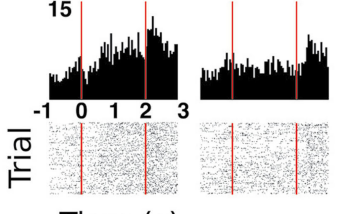

Time (s)

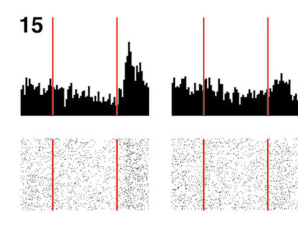

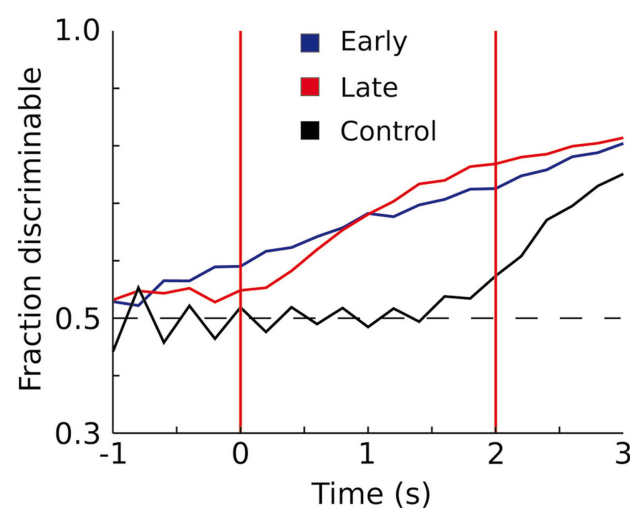

FIGURE 3 | Learning to produce vibratory and ICMS cued reaches.

(A) Improvement in target discrimination performance where somatosensory information was delivered via vibrotactile stimulation. Circles indicate Monkey 1; squares indicate Monkey 2. Filled symbols indicate statistically significant difference from chance performance $(P<0.01)$, open symbols correspond to chance performance. Solid lines indicate sigmoidal fits. (B) Improvement in target discrimination where somatosensory information was delivered via ICMS of Monkey 1. Solid line indicates sigmoidal fit. (C) Improvement in target discrimination in Monkey 1 after a reversal of the association between the ICMS and the response target. Solid line indicates linear fit. (D) Psychometric curve of behavioral performance as ICMS pulse train amplitude was modulated. Solid line indicates sigmoidal fit. (E) Peri-event time histograms and rasters of the activity of four single units from M1 of Monkey 1. Trials are aligned on cue onset. Vertical red lines indicate start and end of the cue interval. Left columns indicate ICMS trials during which the monkey moved to the left; right columns indicate movements to the right. (F) Average linear discriminant analysis predictions of movement direction as a function of time since cue onset show learning-related changes. Blue and red traces indicate averages across early $(n=15)$ and late $(n=15)$ sessions, respectively; control sessions $(n=2)$ are shown in black. Vertical red lines indicate start and end of the cue interval.
In other words, the animal was simply guessing. However, after learning (red trace, Figure 3F), the rise in neuronal prediction occurred subsequent to the instruction period onset and had a steeper slope, indicating a more rapid accumulation of evidence for the reach direction. In control sessions in which no ICMS was presented (black trace, Figure 3F), information about the impending movement was not present in the population activity until $0.5 \mathrm{~s}$ before movement onset. This indicates that in the late 
sessions, the monkey did not make premature guesses. Instead, the evidence suggests that this monkey formed its target choices based on the pattern ICMS.

Real-time, direct brain control was instructed by ICMS in sessions with Monkey 1 (Figure 4B) and by vibrotactile stimulation in sessions with Monkey 2 (Figure 4A). Monkey 1 performed without using the joystick in some sessions (Figure 4C);
Monkey 2 continued to use the joystick (which was needed to produce vibrotactile stimulation). Both monkeys exceeded $70 \%$ correct performance in brain control. Monkey 1 performed above this level in seven sessions of brain control with ICMS. Monkey 2 started by performing at a $55 \%$ correct performance level in brain control with vibrotactile input, and reached a greater than $80 \%$ performance on the seventh session of training.
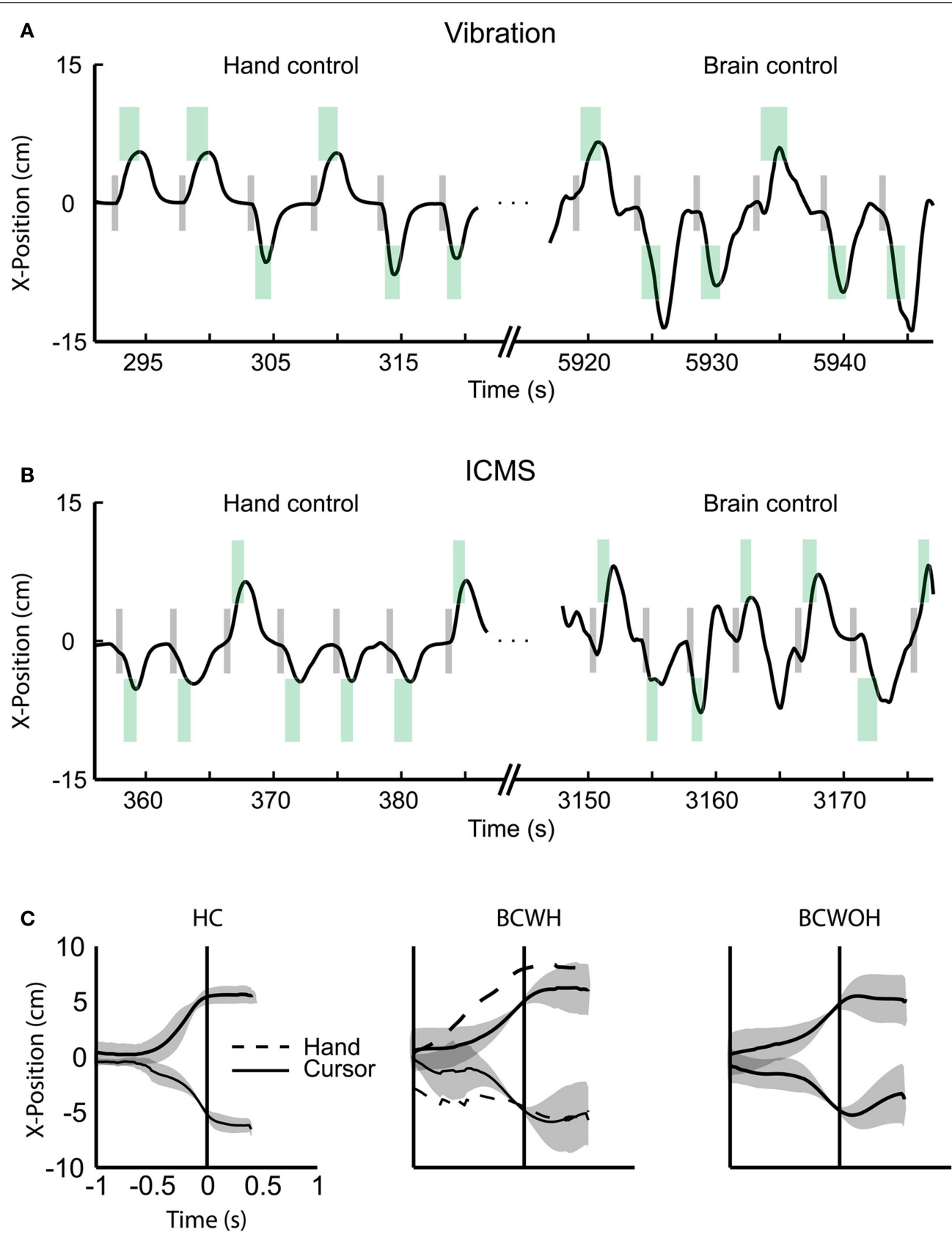

FIGURE 4 | BMI operations. (A) The $x$-component of cursor position during the target choice task, illustrating manual control (left) and BMI control (right) from a single session with Monkey 2. Gray rectangles represent the instruction period; green rectangles represent the location of the correct target for each trial, its onset and offset time (horizontal dimension) and size (vertical dimension). (B) Same as panel A, but depicting a session with Monkey 1. (C) Average movements made by Monkey 1 in the left and right directions for hand control, $\mathrm{HC}$ (left), BMI control with hand movements, BCWH (middle) and BMI control without hand movements, $\mathrm{BCWOH}$ (right). Trajectories are aligned on the time of target acquisition. Solid trace indicates mean cursor movements and dashed trace indicates mean hand movements. Shaded region is standard deviation of cursor movements. 


\section{DISCUSSION}

Altogether, these experiments demonstrate that somatosensory input can be incorporated in BMIs using either vibrotactile stimulation or ICMS of S1. This type of recursive somatosensory input can be considered a form of feedback because the stimulus was contingent on cursor position. The notion that electrical stimulation of cortical areas may reproduce physiological sensations dates back to the pioneering studies of Penfield (Penfield and Boldrey, 1937). More recently, ICMS of S1 proved to be efficient to reproduce temporal (Romo et al., 1998) and spatiotemporal (Fitzsimmons et al., 2007) parameters of somatosensory inputs. Our findings are consistent with our previous demonstration that spatiotemporal patterns of ICMS delivered through chronically implanted multielectrode arrays are viable over long intervals, leading to steady improvement of the behaviors instructed by ICMS (Fitzsimmons et al., 2007).

As in our previous study (Fitzsimmons et al., 2007), we did not observe an immediate ability to interpret ICMS applied to S1 in the monkeys previously overtrained with vibrotactile stimuli, which indicates a substantial difference between the sensations evoked by skin vibration and ICMS. This result is different from the description of Romo et al. (1998) who reported almost immediate learning with ICMS of S1. One explanation for this discrepancy is the specific S1 subdivision implanted. Romo et al. (1998) implanted area $3 \mathrm{~b}$, which is upstream to area 1 implanted in our study. Additionally, their experiment included a number of initial test sessions during which learning to discriminate ICMS could have occurred.

When Monkey 1 finally did start learning the ICMS cue, its learning took a very rapid time course (Figure 3B). One possible interpretation is that the sensations evoked by ICMS of area 1 were quite unlike skin vibration. Another explanation is that no sensations were initially evoked and that repeated exposure to ICMS was required for any sensation to develop. Further experiments will be required to better understand factors involved in the development of sensation due to S1 ICMS. Although ICMS of area 1 may not initially evoke sensations very similar to skin vibration, as follows from our present study and previous work (Fitzsimmons et al., 2007), this does not at all preclude its use in BMI applications.

The action of S1 ICMS was not as trivial as stimulating M1 through the direct projections from S1 to M1. M1 recordings did not reveal any ICMS-evoked responses per se in this cortical area. The presence of such evoked responses would be consistent with the animal's behavioral response being mediated by a simple detection-like signal, caused by ICMS, migrating from S1 to M1. Instead, we observed the occurrence of a gradual build up of preparatory neuronal activity that did not merely mirror the constant frequency of ICMS (Figures 3E,F). Moreover, there was a $33 \%$ reaction time difference observed between low ICMS current amplitudes $(<20 \mu \mathrm{A})$ and high ICMS current amplitudes $(>20 \mu \mathrm{A})$ for correction trials in which the monkey knew the correct response. This difference provides further evidence that the monkey was attending to the ICMS cue. Finally, the monkey successfully learned an ICMS-reach association reversal from a target contralateral to the stimulated hemisphere to a target ipsilateral to the stimulus (Figure 3C). This would not happen if the monkey simply reached toward the hemifield contralateral to M1 which was excited by the projections from $\mathrm{S} 1$.

The second monkey was unable to learn to discriminate ICMS of PP, even after 30 daily sessions. This result mirrored our previous observation in an owl monkey that was unable to learn to discriminate PP stimulation but later learned to discriminate ICMS delivered to S1 (Fitzsimmons et al., 2007). Therefore, it is conceivable that PP cannot be used for this type of sensory instruction or that use of PP may require much longer training or require different parameters. This result suggests that for ICMS to instruct target selection, it may not be sufficient to simply inject current to any part of the cortical network that controls reaching movements, for example PP. Stimulation of primary sensory areas of the cortex (and possibly thalamus) appears to be most effective for sensory substitution, as follows from our present study, as well as previous studies by us (Fitzsimmons et al., 2007) and others (Richer et al., 1993).

Several recent studies reported adding vibrotactile sensory channels to EEG-based BMIs (Chatterjee et al., 2007; Cincotti et al., 2007). While sensory substitution using haptic devices (VidalVerdu and Hafez, 2007) is clearly an important development that will benefit patients, sensorizing BMIs with ICMS is potentially capable of surpassing sensory substitution. Indeed, by using ICMS to directly target the cortical areas deprived of sensory input after neurological injury, disease or limb loss, we endeavor to directly use intact brain functionality rather than delegating sensory processing to other structures.

The present demonstration of a BMI instructed by ICMS has brought together two experimental paradigms that were previously studied in isolation: real-time BMI control based on neuronal ensemble recordings from cortical motor areas (Wessberg et al., 2000; Serruya et al., 2002; Taylor et al., 2002; Carmena et al., 2003; Moritz et al., 2008; Velliste et al., 2008) and ICMS of S1 as a means to evoke somatic sensations (Romo et al., 1998; Talwar et al., 2002; Butovas and Schwarz, 2007; Fitzsimmons et al., 2007; Huber et al., 2008; London et al., 2008). Our results show that, with training, monkeys can learn to select their reaching movements using somatosensory cues, and one monkey reported here learned to utilize S1 ICMS to mediate BMI-controlled reaching movements. We have established the feasibility of moving from a brain-machine interface to a brain-machine-brain interface in which artificial actuators and brain tissue are directly connected in a bidirectional way. These results lead us to suggest that in the future, ICMS delivered through chronically implanted multielectrode arrays may be incorporated into many BMI paradigms and used for delivering artificial sensory feedback directly to the subject's brain.

\section{ACKNOWLEDGEMENTS}

We are grateful to Gary Lehew for assistance in building the experimental setup; Zheng Li for contributions to the BMI software; Dragan Dimitrov, Laura Oliveira and Aaron Sandler for surgical assistance; Weiying Drake, Tamara Phillips and Benjamin Grant for experimental support; Craig Henriquez for comments on the manuscript and Susan Halkiotis for administrative assistance. This work was supported by DARPA award N66001-06-C-2019 and USAMRMC award W81XWH-08-2-0119 to MALN. 


\section{REFERENCES}

Butovas, S., and Schwarz, C. (2007). Detection psychophysics of intracortical microstimulation in rat primary somatosensory cortex. Eur. J. Neurosci. $25,2161-2169$.

Carmena, J. M., Lebedev, M. A., Crist, R. E., O’Doherty, J. E., Santucci, D. M., Dimitrov, D. F. Patil, P. G., Henriquez, C. S., and Nicolelis, M. A. (2003). Learning to control a brain-machine interface for reaching and grasping by primates. PLoS Biol. 1, E42. doi: 10.1371/journal.pbio.0000042.

Chatterjee, A., Aggarwal, V., Ramos, A., Acharya, S., and Thakor, N. V. (2007). A brain-computer interface with vibrotactile biofeedback for haptic information. J. Neuroeng. Rehabil. 4, 40 .

Cincotti, F., Kauhanen, L., Aloise, F., Palomaki, T., Caporusso, N., Jylanki, P., Babiloni, F., Vanacker, G., Nuttin, M., Marciani, M. G., Del, R. M. J., and Mattia, D. (2007). Preliminary experimentation on vibrotactile feedback in the context of mu-rhythm based BCI. Conf. Proc. IEEE Eng. Med. Biol. Soc. 2007, 4739-4742.

Crapse, T. B., and Sommer, M. A. (2008). Corollary discharge across the animal kingdom. Nat. Rev. Neurosci. 9, 587-600.

Dagnelie, G. (2008). Psychophysical evaluation for visual prosthesis. Annu. Rev. Biomed. Eng. 10, 339-368.

Dobelle, W. H., Mladejovsky, M. G., and Girvin, J. P. (1978). Artificial vision for the blind: electrical stimulation of the cortex offers hope for a functional prosthesis. Science 183, 1-39.

Fallon, J. B., Irvine, D. R., and Shepherd, R. K. (2008). Cochlear implants and brain plasticity. Hear. Res. 238, 110-117.

Fetz, E. E. (2007). Volitional control of neural activity: implications for brain-computer interfaces. J. Physiol. 579, 571-579.

Fisher, R. A. (1936). The use of multiple measurements in taxonomic problems. Ann. Eugen. 7, 179-188.
Fitzsimmons, N. A., Drake, W., Hanson, T. L., Lebedev, M. A., and Nicolelis, M. A. (2007). Primate reaching cued by multichannel spatiotemporal cortical microstimulation. J. Neurosci. 27, 5593-5602.

Hanks,T.D.,Ditterich,J., andShadlen, M. N. (2006). Microstimulation of macaque area LIP affects decision-making in a motion discrimination task. Nat. Neurosci. 9, 682-689.

Hatsopoulos, N., Joshi,J., and O'Leary, J. G. (2004). Decoding continuous and discrete motor behaviors using motor and premotor cortical ensembles. J. Neurophysiol. 92, 1165-1174.

Haykin, S. (2002). Adaptive Filter Theory, 4th Edn. Upper Saddle River, NJ, Prentice Hall.

Huber, D., Petreanu, L., Ghitani, N., Ranade, S., Hromadka, T., Mainen, Z., and Svoboda, K. (2008). Sparse optical microstimulation in barrel cortex drives learned behaviour in freely moving mice. Nature 451, 61-64.

James, T. W., Kim, S., and Fisher, J. S. (2007). The neural basis of haptic object processing. Can. J. Exp. Psychol. 61, 219-229.

Lebedev, M. A., Carmena, J. M., O'Doherty, J. E., Zacksenhouse, M., Henriquez, C. S., Principe, J. C., and Nicolelis, M. A. (2005). Cortical ensemble adaptation to represent velocity of an artificial actuator controlled by a brain-machine interface. J. Neurosci. 25, 4681-4693.

Lebedev, M. A., and Nicolelis, M. A. (2006). Brain-machine interfaces: past, present and future. Trends Neurosci. 29, 536-546.

London, B. M., Jordan, L. R., Jackson, C. R., and Miller, L. E. (2008). Electrical stimulation of the proprioceptive cortex (area 3a) used to instruct a behaving monkey. IEEE Trans. Neural Syst. Rehabil. Eng. 16, 32-36.

Merzenich, M. M., Schindler, D. N., and White, M. W. (1974). Feasibility of multichannel scala tympani stimulation. Laryngoscope 84, 1887-1893.

Moritz, C. T., Perlmutter, S. I., and Fetz, E. E. (2008). Direct control of paralysed muscles by cortical neurons. Nature 456, 639-642.

Nicolelis, M. A. (2001). Actions from thoughts. Nature 409, 403-407.

Nicolelis, M. A. (2003). Brain-machine interfaces to restore motor function and probe neural circuits. Nat. Rev. Neurosci. 4, 417-422.

Nicolelis, M. A., Dimitrov, D. Carmena, J. M., Crist, R., Lehew, G. Kralik, J. D., and Wise, S. P. (2003). Chronic, multisite, multielectrode recordings in macaque monkeys. Proc. Natl. Acad. Sci. U.S.A. 100, 11041-11046.

Penfield, W., and Boldrey, E. (1937) Somatic motor and sensory representation in the cerebral cortex of man as studied by electrical stimulation. Brain 60, 389-443.

Ribot-Ciscar, E., Bergenheim, M. Albert, F., and Roll, J. P. (2003). Proprioceptive population coding of limb position in humans. Exp. Brain Res. 149, 512-519.

Richer, F., Martinez, M., Robert, M., Bouvier, G., and Saint-Hilaire, J. M. (1993). Stimulation of human somatosensory cortex: tactile and body displacement perceptions in medial regions. Exp. Brain Res. 93 173-176.

Romo, R., Hernandez, A., Zainos, A., and Salinas, E. (1998). Somatosensory discrimination based on cortical microstimulation. Nature 392, 387-390.

Schwartz, A. B., Cui, X. T., Weber, D. J., and Moran, D. W. (2006). Brain-controlled interfaces: movement restoration with neural prosthetics. Neuron 52, 205-220.

Serruya, M. D., Hatsopoulos, N. G. Paninski, L., Fellows, M. R., and Donoghue, J. P. (2002). Instant neural control of a movement signal. Nature 416, 141-142.

Talwar, S. K., Xu, S., Hawley, E. S., Weiss, S.A., Moxon, K. A., and Chapin, J. K. (2002). Rat navigation guided by remote control. Nature 417, 37-38.

Taylor, D. M., Tillery, S. I., and Schwartz, A. B. (2002). Direct cortical control of 3D neuroprosthetic devices. Science 296, 1829-1832.

Tkach, D., Reimer, J., and Hatsopoulos, N. G. (2007). Congruent activity during action and action observation in motor cortex. J. Neurosci. 27, 13241-13250.

Velliste, M., Perel, S., Spalding, M. C., Whitford, A. S., and Schwartz, A. B (2008). Cortical control of a prosthetic arm for self-feeding. Nature 453, 1098-1101.

Vidal-Verdu, F., and Hafez, M. (2007). Graphical tactile displays for visuallyimpaired people. IEEE Trans. Neural Syst. Rehabil. Eng. 15, 119-130.

Wessberg, J.,Stambaugh,C.R., Kralik, J. D., Beck, P. D., Laubach, M., Chapin, J. K., Kim, J., Biggs, S. J., Srinivasan, M. A., and Nicolelis, M. A. (2000). Realtime prediction of hand trajectory by ensembles of cortical neurons in primates. Nature 408, 361-365.

Witney, A. G., Wing, A., Thonnard, J. L., and Smith, A. M. (2004). The cutaneous contribution to adaptive precision grip. Trends Neurosci. 27, 637-643.

Conflict of Interest Statement: The authors declare that the research was conducted in the absence of any commercial or financial relationships that could be construed as a potential conflict of interest.

Received: 25 June 2009; paper pending published: 21 July 2009; accepted: 08 August 2009; published online: 01 September 2009.

Citation: O'Doherty JE, Lebedev MA, Hanson TL, Fitzsimmons NA, Nicolelis MAL (2009) A brain-machine interface instructed by direct intracortical microstimulation. Front. Integr. Neurosci. 3:20. doi: 10.3389/neuro.07.020.2009 Copyright (c) 2009 O'Doherty, Lebedev, Hanson, Fitzsimmons, Nicolelis. This is an open-access article subject to an exclusive license agreement between the authors and the Frontiers Research Foundation, which permits unrestricted use, distribution, and reproduction in any medium, provided the original authors and source are credited. 